\section{TBPS-Operationen: Verschiedene Verfahren sind sicher}

A lle beim benignen Prostatasyndrom (BPS) angewandten Operationsverfahren sind ausreichend sicher. Das geht aus Routinedaten der AOK hervor. Es könne keinem der untersuchten Operationsverfahren eine eindeutige Überlegenheit in allen Teilaspekten der Therapie zugesprochen werden, so Dr. med. Christian Gilfrich, Klinikum St. Elisabeth, Straubing, der die Daten des wissenschaftlichen Instituts der AOK vorgestellt hat. Demnach hatten sich im Untersuchungszeitraum von 2008 bis 2013 bundesweit etwa 78.000 Männer einer transurethralen Resektion (TURP), einer Laservaporisation (LVP), einer Laserenukleation (LEP) oder einer offenen Operation unterzogen. Die Häufigkeit der TURP nimmt demnach zwar zugunsten der Laserverfahren ab, nach wie vor werden aber drei Viertel aller Patienten auf diese Weise operiert. LVP und LEP machen inzwischen $13 \%$ der Ein- griffe aus, der Anteil offener Operationen liegt stabil zwischen 8 und $9 \%$.

Die 30-Tage-Sterblichkeitsrate betrug insgesamt 0,3-0,5\%. Die meisten Bluttransfusionen waren erwartungsgemäß nach offenen Operationen erforderlich, die auch sonst die im Vergleich höchsten Komplikationsraten aufwiesen. Rechnet man per multivariater Regressionsanalyse Einflussfaktoren wie Alter, Komorbiditäten und antithrombotische Medikation in den Gruppen heraus, ergibt sich für die offene Operation aber auch die niedrigste Reinterventionsrate, nämlich halb so oft wie mit TURP. Die höchste Reinterventionsrate zeigt dagegen die LVP, nämlich 1,4-mal so oft wie mit TURP. Reinterventionen wegen Blutungen waren mit der LEP 1,3-mal häufiger als mit TURP nötig, dafür seltener mit LVP - 0,76 im Vergleich zur TURP.

„Bei der Laservaporisation ist das Transfusions- und Nachblutungsrisiko geringer, aber die Wahrscheinlichkeit für Reinterventionen erhöht", fasste Gilfrich die Daten zusammen. „Bei der Laserenukleation zeigt sich ein erhöhtes Risiko für Eingriffe wegen Nachblutungen."

Allerdings verwies Gilfrich auch auf die limitierte Aussagekraft der Routinedaten: Sie enthielten keine klinische $\mathrm{Pa}$ rameter, etwa zum Prostatavolumen. Vorbestehende Diagnosen ließen sich nicht von Erkrankungen unterscheiden, die erst im Laufe des Krankenhausaufenthalts aufträten. Zudem könnten funktionelle Parameter nicht verlässlich abgebildet werden. Dennoch ergeben sich aus der Untersuchung Hinweise, wonach Patientenalter, Komorbidität und die Abschätzung der postoperativen Komplikationsrate in die Indikationsstellung zum jeweiligen Operationsverfahren einbezogen werden sollten.

Dr. Thomas Meißner

Vortragssitzung „Komplikationsraten verschiedener Eingriffe bei BPS: eine bundesweite Analyse von AOK-Routinedaten“, C. Gilfrich, DGUKongress, Leipzig, 30. September 2016

\title{
Nierenzellkarzinom: zu intensive Nachsorge?
}

st die Nachsorge von Patienten nach kurativer Lokaltherapie bei klarzelligem Nierenzellkarzinom womöglich $\mathrm{zu}$ intensiv? Prof. Dr. med. Thomas Steiner aus Erfurt verwies auf eine aktuelle Studie, wonach bei 1.400 Niedrigrisikopati- enten innerhalb von drei Jahren lediglich acht Rezidive oder Metastasen gefunden worden waren. Auf insgesamt 5.762 bildgebende Untersuchungen kamen nur sechs behandelte Patienten. Statistisch wären fast 1.000 Computertomografien

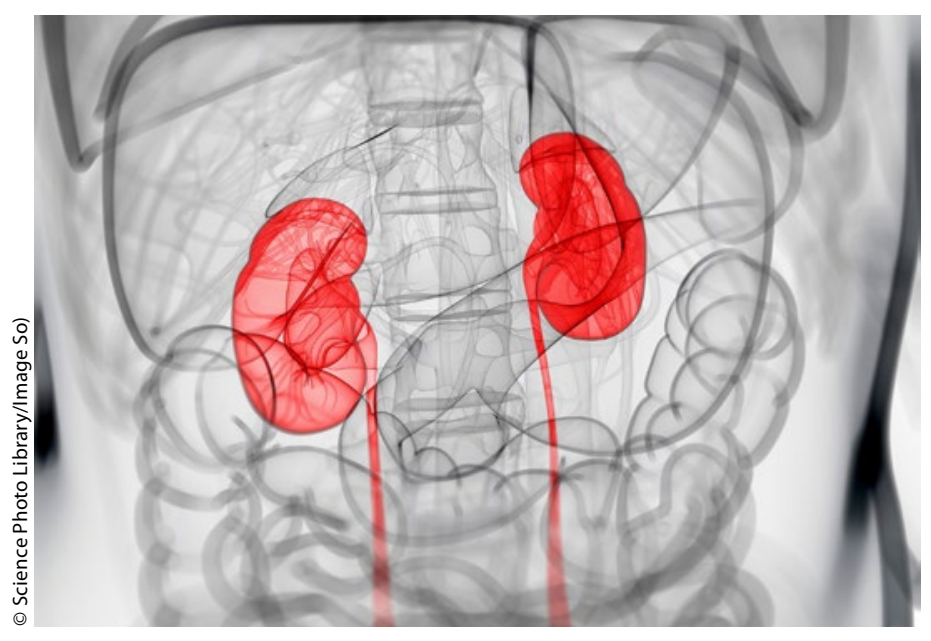

Bei bestimmten Patienten mit einem Nierenzellkarzinom sollte die Nachsorge reduziert werden. erforderlich, um ein behandlungsbedürftiges Rezidiv zu finden [Feuerstein MA. World J Urol. 2016; 34:949-53]. „Da stellt sich natürlich die Frage nach Effektivität und Effizienz", so Steiner, der einer der Autoren der aktuellen S3-Leitlinie zum Nierenzellkarzinom ist. „Es geht nicht nur um die Kosten, sondern auch um die Strahlenbelastung der Patienten." Zudem sei nicht gesichert, inwiefern durch die aktuellen Nachsorgeempfehlungen die Gesamtüberlebenszeit der Patienten tatsächlich verlängert werde. Bei Patienten mit intermediärem und hohem Risiko sei festgestellt worden, dass früh auftretende Metastasen in der Regel nur palliativ behandelt werden können, später auftretende Rezidive dagegen oft kurativ. „Es könnte sinnvoll sein, bei diesen Patienten die Nachsorge in den ersten Jahren zu minimieren und erst nach zwei Jahren mit einer intensiven Nachsorge zu beginnen“, betonte Steiner. Dr. Thomas Meißner

Forumssitzung „Kritische Diskussion der Nachsorgestrategien nach kurativer Lokaltherapie des Nierenzellkarzinoms", T. Steiner, DGU-Kongress, Leipzig, 29. September 2016 